\title{
Bullous Lesions on a Skin Graft Donor Site
}

\author{
Alissa K. Orvis, BS, Volba Ihnatsenka, MD, and Robert L. Hatch, MD, MPH
}

Patients with bullous lesions often present to family physicians. In many cases, the diagnosis is readily apparent based on a focused history and examination. We report a case in which the correct diagnosis, bullous pemphigoid (BP), was obscured by an unusual presentation. Although some lesions were typical of BP, most were clustered on a recent skin graft donor site and some were hemorrhagic. A review of recent family medicine literature revealed one article discussing $\mathrm{BP},{ }^{1}$ but neither this article nor 3 dermatology textbooks ${ }^{2-4}$ mentioned this presentation. However, further literature review revealed that this presentation is actually well described and fits nicely with current understanding of the etiology of BP. The review also revealed an important recent change in $\mathrm{BP}$ management: the emergence of ultra-high-potency topical steroids as a first line therapy. As recently as 6 years ago, oral steroids were the standard approach for treating most BP patients. The following case provides an opportunity to review recent advances in the understanding and management of $\mathrm{BP}$, a condition most family physicians can expect to encounter. ${ }^{3}$ The discussion also addresses the differential diagnosis of bullous lesions and the need for special punch biopsy specimen media if BP is suspected.

\section{Case Reports}

A 53-year-old woman presented with painless blisters of several days' duration. One month before she sustained a third-degree burn of the right foot requiring a split-thickness skin graft. Her blisters were disproportionately concentrated on the skin

This article was externally peer reviewed.

Submitted 9 February 2008; revised 14 April 2008; accepted 17 April 2008.

From the Department of Community Health and Family Medicine, University of Florida College of Medicine, Gainesville.

Funding: none.

Conflict of interest: none declared.

Corresponding author: Robert L. Hatch, MD, MPH, Department of Community Health and Family Medicine, University of Florida College of Medicine, Box 100222, Gainesville, FL 32610 (E-mail: hatch@ufl.edu). graft donor site (Figure 1) but were also present on the left shin (Figure 2). She denied fever and itching and complained only of chronic foot pain ever since skin graft surgery. She denied applying any topical products to the donor site or her legs and denied any recent change in skin care products. The patient had never experienced a similar blistering episode.

Her recent medical history included a hospital admission 2 weeks earlier for aspiration pneumonia. Recovery was uneventful apart from Clostridium difficile colitis caused by piperacillin/tazobactam, which was successfully treated with metronidazole. Medical history included insulin-dependent diabetes, chronic renal insufficiency, coronary artery disease, hypertension, and cerebrovascular accident with mild residual right-sided weakness. Medications included oxycodone/acetaminoiphen; lansoprazole; aspirin; atorvastatin; clopidogrel; metoprolol; venlafaxine; albuterol; gabapentin; insulin; Culturelle (an OTC pro-biotic; Amerifit Brands, Inc., Cromwell, CT); and metronidazole.

Vital signs were normal during physical examination. The skin graft donor site contained multiple tense bullae with hemorrhagic contents (Figure 1). Similar bullae with clear fluid were seen on the left anterior shin (Figure 2). The bullae were oval to round in shape, 1.5 to $4 \mathrm{~cm}$ in diameter, tense, and painless to the touch. There were no lesions in the oral cavity. Gentle pressure applied to the blisters did not cause them to spread outwards, thus yielding a negative Nikolsky's sign. Further physical examination was noncontributory. Although the left shin lesions were characteristic of BP, the clustering of lesions on the donor site led to uncertainty about the diagnosis. After some debate, a punch biopsy of perilesional skin was obtained and treatment was initiated with oral prednisone $40 \mathrm{mg}$ daily for presumptive pemphigoid. In a matter of days, current lesions began to heal and new lesions stopped appearing. Punch biopsy revealed linear deposit of C3 along the basement membrane, confirming a diagnosis of $\mathrm{BP}$. 


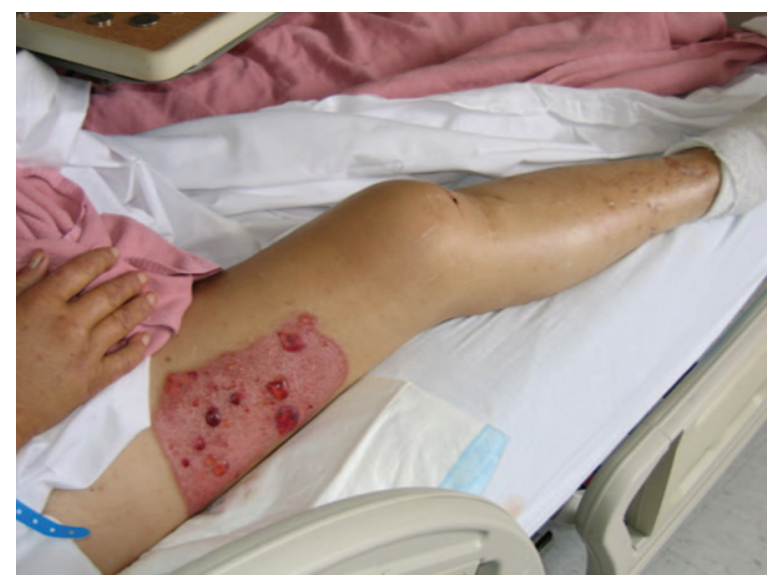

Figure 1. Bullous lesions clustered on a recent skingraft donor site. Note that the bullae are tense and most contain hemorrhagic fluid.

\section{Discussion}

$\mathrm{BP}$ is the most common autoimmune blistering disorder. It arises equally in men and women, usually between the ages of 60 and $80 .{ }^{1}$ The disease has a variable course. Milder cases may be fairly benign and self-limited but may last several years. ${ }^{1}$ However, in older and debilitated patients, untreated generalized disease has a 1-year mortality rate of $20 \%$ to $45 \% .^{5}$ Thus, although BP is not as severe as pemphigus vulgaris, it can be quite serious. Although BP is not common, ${ }^{6}$ most family physicians can expect to encounter it, ${ }^{3}$ especially if they care for large numbers of elderly patients. Even if $\mathrm{BP}$ is never encountered, a working knowledge of the condition is very helpful when evaluating patients with bullous lesions.

$\mathrm{BP}$ is an autoimmune disease in which the body "attacks" hemidesmisomes, the structures that bind cells together at the basement membrane of the dermoepidermal junction. ${ }^{6}$ Autoantibodies react with antigens BP 180 and BP 230 on these hemidesmisomes. ${ }^{6-8}$ This activates the complement pathway, creating subepidermal blisters via local inflammation. ${ }^{7}$ Because BP blisters are subepidermal, the blisters have a thick upper layer and are usually quite large, tense, and reluctant to rupture. They are often pruritic and may be preceded by erythema or urticaria. ${ }^{1}$ The fluid in the lesions is usually clear but it may be hemorraghic, ${ }^{1}$ especially in older lesions ${ }^{3}$ and lesions that arise on sites of previous trauma. ${ }^{7}$ Lesions may be generalized or localized. The lower legs are often the first site of manifestation. ${ }^{4}$ The oral mucosa may be involved, but much less commonly than in pemphigus vulgaris. ${ }^{1}$

Lesions are known to show a predilection for, or localize solely to, sites of recent dermatologic trauma. ${ }^{7}$ Three cases of BP arising in spilt-thickness skin grafts and/or their donor sites have been reported. ${ }^{7,9,10} \mathrm{BP}$ has been reported in other surgical sites as well. ${ }^{11}$ This represents a form of "koebnerization," the phenomenon of dermatologic lesions appearing at a site of injury. ${ }^{12}$ Anderson ${ }^{11}$ describes 3 possible scenarios occurring in patients who present with BP localized to an area of prior trauma: (1) the blisters remain localized to the original site of trauma; (2) lesions are initially localized then become generalized; (3) localized lesions develop in patients with pre-existing generalized disease.

Current theories about the molecular basis for $\mathrm{BP}$ are consistent with the observation that $\mathrm{BP}$ can localize to areas of skin trauma. It is hypothesized that local injury unmasks BP antigens, increasing the likelihood of an autoimmune attack. ${ }^{9}$ Another hypothesis suggests that during the healing process, $\mathrm{BP}$ antigens become more available for binding, leading to attack. ${ }^{9}$ This hypothesis may explain the several-week delay often observed ${ }^{9-11}$ between injury and blister appearance, because the healing process is fairly lengthy. These theories also suggest a rationale for the presence of both local and

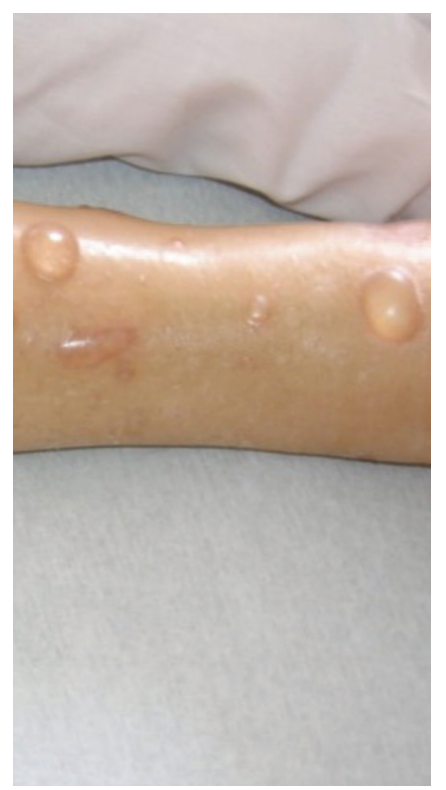

Figure 2. Bullous lesions on the left ankle. These lesions are more typical of bullous pemphigoid (tense with clear fluid). 
distant bullae. A disruption exposing the antigen could allow for autoimmune attack, which, with natural amplification of the immune response, could generalize to other "normal" areas (Anderson's second scenario). This may explain the presence of lesions on both the donor site and normal skin, as in the case described above. Some cases of $\mathrm{BP}$ are thought to be drug induced. One class that has been implicated is oral beta-blockers, ${ }^{13}$ and it is possible that metoprolol contributed to the development of BP in this patient.

The diagnosis of BP is typically confirmed via punch biopsy and direct immunofluorescence testing. Perilesional skin is the optimal site for biopsy. The biopsy specimen must be placed in a special medium (such as Michel's) because formalin interferes with immunofluorescence testing. Direct-immunofluorescence shows linear deposits of immunoglobulin $\mathrm{G}$ and/or C3 at the basement membrane zone. ${ }^{6}$ Light microscopy reveals histiocyte, eosinophil, and lymphocyte infiltration of the dermis. ${ }^{1}$ Serum tests can detect circulating immunoglobulin $\mathrm{G}$ autoantibodies via indirect immunofluorescence to the basement membrane; however, this test is positive in only $70 \%$ of cases and the titer does not correlate with disease severity. ${ }^{4}$

The differential diagnosis of bullous lesions includes burns; contact dermatitis; impetigo; photodermatitis; viral illnesses such as herpes and varicella, bullous pemphigoid; and other autoimmune bullous diseases. ${ }^{1,14}$ Contact dermatitis must be considered in a presentation similar to our case. Patients often apply lotions, ointments, and other substances that may cause contact dermatitis to wounds. ${ }^{15}$ This may present as pruritic vesicular or bullous lesions on an erythematous base. Asking about topical treatments can help identify potential causes. Photodermatitis would be more likely to appear in areas of skin trauma because much of the skin's protective elements are lost. Bacterial infection should also be suspected in cases similar to ours. Impetigo caused by secondary infection of a healing wound may present as bullae. ${ }^{14}$ Other autoimmune blistering disorders may be the culprit, including pemphigus vulgaris, paraneoplastic pemphigus, cicatricial pemphigoid, dermatitis herpetiformis, and linear immunoglobulin A dermatosis. ${ }^{1}$ Although these autoimmune conditions are generally more serious than $\mathrm{BP}$, with the exception of dermatitis herpetitformis, they are rare.
The goal of treating BP is to prevent further blister formation and promote the healing of current lesions, using the least amount of medication possible. Optimal treatment is controversial, and treatment plans must be individualized. Patients generally respond quickly to systemic corticosteroids, but this must be balanced against adverse effects. In fact, oral corticosteroids at higher doses actually increase the mortality rate in patients with severe BP. ${ }^{16}$ New research suggests that ultrahigh-potency topical corticosteroid treatment may achieve similar disease control while avoiding the problems associated with oral corticosteroids. ${ }^{6}$ Kirtschig and Khumalo ${ }^{6}$ recommend the following approach, which is also supported by a $2005 \mathrm{Co}-$ chrane review ${ }^{17}$ : for localized disease, topical clobetasol proprionate cream; for mild to moderate generalized disease, oral tetracyclines (with or with out niacinamide) plus topical steroids. If this regimen is not effective, they recommend lower dose oral prednisone (no higher than $0.5 \mathrm{mg} / \mathrm{kg} /$ day). Higher doses are reserved for cases that do not respond after 4 to 8 weeks. Other agents are also available and may be considered. ${ }^{6}$

Patients presenting with blisters in areas of previous trauma can present a diagnostic conundrum for family physicians. Localized BP is often not considered as a possible culprit and proper treatment may unknowingly be delayed or never provided. Awareness of the tendency of BP to arise in scars is crucial because this triggers the appropriate diagnostic procedures ultimately leading to the correct, and generally quite effective, treatment.

\section{References}

1. Bickle KM, Roark TR. Autoimmune bullous dermatoses: a review. Am Fam Physcian 2002;65:1861-70.

2. Nousair HC, Dnhalt GJ. Autoimmune bullous dieseaes, in cutaneous medicine. In: Provost TT, Flynn JA (eds). Hamilton, Ontario: BC Decker; 2001.

3. du Vivier A. Atlas of clinical dermatology. London, UK: WB Saunders; 1986.

4. Wolff K, Johnson RA, Suurman D. Color atlas and synopsis of clinical dermatology, 5th ed. New York: McGraw-Hill; 2005:106-8.

5. Joly P, Lok C, Saiag P, et al. Prediction of survival for patients with bullous pemphigoid. Arch Dermatol 2005;141:691-8.

6. Kirtschig G, Khumalo NP. Management of bullous pemphigoid. Am J Clin Dermatol 2004;5:319-26.

7. Hafejee A, Coulson IH. Localized bullous pemphi- 
goid 20 years after split skin grafting. Clin Exp Dermatol 2005;30:187-8.

8. Mueller S, Klaus-Kovtun V, Stnaley JR. A 230-kD protein is the major bullous pemphigoid antigen. J Invest Dermatol 1989;93:33-8.

9. Ghura HS, Johnston GA, Milligan A. Development of bullous pemphigoid after split-skin grafting. Br J Plastic Surg 2001;54:447-62.

10. McGrath J, Black M. Split skin grafting and bullous pemphigoid. Clin Exp Dermatol 1991;16:72-3.

11. Anderson CK, Mowad CM, Goff ME, Pelle MT. Bullous pemphigoid arising in surgical wounds. Br J Dermatol 2001;145:670-2.

12. Weiss G, Shemer A, Trau H. The Koebner phenomenon: review of the literature. J Eur Acad Dermatol Venereol 2002;16:241-8.
13. Perry A, Sparling JD, Pennington M. Bullous pemphigoid following therapy with an oral beta-blocker. J Drugs Dermatol 2005;4:746-8.

14. Davies H, Oudit D, Ellaban M, Juma A. Post-burn blistering: a possible diagnostic dilemma. Burns 2004;30:291-2.

15. Jacob S, Steele T. Allergic contact dermatitis: early recognition and diagnosis of important allergens. Derm Nursing 2006;18:433-46.

16. Joly P, Roujeau JC, Benichou J, et al. A comparison of oral and topical corticosteroids in patients with bullous pemphigoid. N Engl J Med 2002;346:321-7.

17. Khumalo N, Kirtschig G, Middleton P, Hollis S, Wojnarowska F, Murrell DF. Interventions for bullous pemphigoid. Cochrane Database Syst Rev 2005; (3):CD002292. 